\title{
Estudio de Índices Faciales en Alumnos de la Universidad de La Frontera, Chile
}

\author{
Facial Indices Study in Students of the Universidad de La Frontera, Chile
}

"Bustamante, F.; "Olave, E. \& **Binvignat, 0.

\begin{abstract}
BUSTAMANTE, F.; OLAVE, E. \& BINVIGNAT, O. Estudio de índices faciales en alumnos de la Universidad de La Frontera, Chile. Int. J. Morphol., 29(4):1335-1340, 2011.

RESUMEN: Las características faciales de los seres humanos le otorgan una individualidad especial, que permiten identificarlos dentro de un grupo determinado, así como también grupos de personas tienen características que le diferencian de otros grupos. Con el fin de determinar características faciales de nuestra población, se estudiaron dos índices faciales, en individuos adultos (18-35 años), de ambos sexos, estudiantes de la Universidad de La Frontera, Chile. Los índices investigados fueron el Facial Superior (IFS), que relaciona altura morfológica de la parte superior de la cara con el diámetro bicigomático máximo y el índice nasal (IN), que relaciona altura del segmento nasal y el ancho nasal. El promedio del IFS fue de 46,8 (DS 4,0), con límites máximo de 62,1 y mínimo de 38,0, clasificando a los individuos estudiados como euriónicos (cara ancha) en $41 \%$ de los casos, como hipereuriónicos (cara muy ancha) en 35\%, como mesenos (cara mediana) en $23 \%$ y como Leptenos (cara angosta) en $1 \%$. El promedio del IN fue de 43,5 (DS 5,1), con límites máximo de 86,2 y mínimo de 50,2, clasificando a los individuos como leptorrinos (nariz angosta) en 77,4\% de los casos, como mesorrinos (nariz mediana) en 17,4\% y como camerrinos (nariz ancha) en un 1,7\%. Los resultados obtenidos muestran que la mayoría de nuestra población es de cara ancha y de nariz delgada, datos que complementarán el conocimiento de las características antropométricas de nuestra región.
\end{abstract}

PALABRAS CLAVE: Índice Facial; Índice Nasal; Adulto Joven; Chile.

\section{INTRODUCCIÓN}

Los seres humanos guardan entre sí las semejanzas que los reunen en una especie determinada, pero presentan grandes diferencias entre ellos mismos, de tal modo que puede asegurarse que no existen dos exactamente iguales. Esto se comprueba tanto en su aspecto exterior como en su medio interno, encontrando diferencias en la anatomía de órganos y tejidos.

Al existir una visible y marcada individualización en lo morfológico, es forzoso incluir que parecidas diferencias han de existir en las funciones y en la psiquis, desarrollada en el más alto grado en la especie humana, donde se encuentra su máxima expresión, la personalidad individual (Oliveros et al., 2006).

Desde el punto de vista anatómico y antropológico, la cabeza es una parte de nuestro cuerpo en donde se manifiestan de manera más expresiva las diferencias morfológicas que caracterizan a los grupos étnicos. Las características faciales de los seres humanos le otorgan una individualidad especial, que permite identificarlo dentro de un grupo determinado, así como también grupos de personas tienen características que le diferencian de otros grupos que habitan el planeta.

También se ha descrito que el dimorfismo sexual parece estar influido por factores culturales, nutricionales y medioambientales, los cuales no afectan al crecimiento y al tamaño de ambos sexos por igual (Stini,1982 y Bogin \& Collier, 1993).

Dentro de la antropología física, se pueden registrar medidas que permiten caracterizar partes y estructuras del cuerpo en los individuos, pudiendo con ello agrupar a los mismos de acuerdo a esas características métricas. Martin \& Saller (1957) en su clásico y monumental "Lehrbuch der Anthropologie", ofrece una visión de la tendencia que se denomina tradicional, o sea, de dar en Antropología Física la mayor importancia a la parte métrica.

\footnotetext{
"Facultad de Medicina, Universidad de La Frontera, Temuco, Chile.

**Universidad Autónoma de Chile, Talca.
} 
Basados en lo anterior, esta investigación ha tenido como objetivo registrar medidas como la altura morfológica de la parte superior de la cara, el diámetro bicigomático máximo, la altura del segmento nasal y la distancia del ancho nasal, y, determinar los índices facial y nasal en un grupo de alumnos de la Universidad de La Frontera, de la Ciudad de Temuco, Chile, complementando así el conocimiento de las características faciales de personas de nuestra población.

\section{MATERIAL Y MÉTODO}

La unidad de estudio fue de 115 individuos vivos, adultos jóvenes, de ambos sexos, estudiantes universitarios de la Facultad de Medicina, Carrera de Odontología, que cursaban $1^{\circ}$ y $2^{\circ}$ año, durante el $2^{\circ}$ semestre Académico del año 2008, en la Universidad de La Frontera, Temuco, Chile. De ellos siete se autodefinieron y consideraron de etnia mapuche, de los cuales todos tenían al menos un apellido mapuche, 3 individuos de sexo femenino y 4 de sexo masculino, lo que corresponde al $6 \%$ de la muestra. Para el registro de medidas se utilizó material de antropometría $a d$ hoc. Las variables consideradas para esta investigación fueron las siguientes:

Altura morfológica de la parte superior de la cara (V1). Distancia directa desde el nasion hasta el prostion;

Diámetro bicigomático máximo (V2). El ancho máximo entre los puntos cefalométricos zigion derecho e izquierdo; Altura del segmento nasal. (V3). Longitud entre el nasion y el subnasion;

Distancia del ancho nasal (V4). El ancho máximo entre los márgenes alares más laterales, dondequiera que se encuentre.

Los índices estudiados están basados en Martin \& Saller (1957) y Pospísil (1985):

Índice facial superior: Relaciona los dos diámetros descritos como V1 y V2:

V1 Altura morfológica superior de la cara: Nasion - prostion (N-Pr) V2 Diámetro bicigomático máximo: Zigion - zigion (Zy-Zy)

Se calcula multiplicando V1 por 100 y dividiendo por V2.

$$
\mathrm{IFS}=(\mathrm{N}-\mathrm{Pr} \mathrm{x} 100 / \mathrm{Zy}-\mathrm{Zy}) \text {. }
$$

Estas variables permiten clasificar a los individuos por su cara, en cinco categorías:

Promedios Hipereuriónico (cara muy ancha) Hasta 44,9

Euriónico (cara ancha) 45,0 - 49,9

Meseno (cara mediana) 50,0 - 54,9

Lepteno (cara angosta) 55,0 - 59,9

Hiperlepteno (cara muy angosta) 60,0 - x
Índice nasal. Relaciona dos diámetros descritos como variables V3 y V4, donde se considera como:

V3 Distancia del ancho nasal Alar - Alar (Al-Al)

V4 Altura del segmento nasal Nasion - Nasioespinal (N-Ns)

Se calcula multiplicando V3 por 100 y dividiendo por V4.

$$
\mathrm{IN}=(\mathrm{Al}-\mathrm{Al} \times 100 / \mathrm{N}-\mathrm{Ns}) \text {. }
$$

Estas variables permiten clasificar a los individuos por su nariz, en cinco categorías:

Hiperleptorrino (nariz muy angosta) $\mathrm{x}-54,9$

Leptorrino ( Nariz angosta) $\quad 55,0-69,9$

Mesorrino (nariz mediana) 70,0 - 84,9

Camerrino (nariz ancha) 85,0 - 99,9

Hipercamerrino (nariz muy ancha) $100-\mathrm{x}$

Los valores registrados se sometieron a un análisis estadístico con programa S.A.S.(SAS System for DEC OSFI Versión 9.2).

\section{RESULTADOS}

La distribución de los promedios de la altura morfológica de la parte superior de la cara, según el sexo, se encuentran expresados en la Tabla I.

Los promedios del diámetro bicigomático máximo (Zy-Zy) según sexo, se muestran en la tabla II.

Con respecto al índice facial superior (IFS), el promedio de IFS fue de 46,8 (DS de 4,0) lo que determinó una población euriónica. El valor mínimo fue de 38,0 (1 persona) clasificándolo como hipereuriónico. Se encontraron 3 individuos con valores bajo 40, lo que corresponde a un $3,5 \%$. El IFS máximo fue de 62,1 (un individuo), clasificándolo como hiperlepteno. La distribución general del IFS en la población estudiada se muestra en la Tabla III.

De los 40 individuos hipereuriónicos, hubo 20 de sexo femenino y 20 de sexo masculino (17,4\% cada grupo), donde en los primeros uno de ellos era de etnia chileno-mapuche $(0,9 \%)$ y en los segundos hubo 2 individuos con esta característica 1,8\%). En el grupo de individuos euriónicos (47), 25 fueron de sexo femenino $(21,8 \%)$ y 22 del sexo masculino $(19,1 \%)$, donde en los primeros hubo un individuo de etnia chileno-mapuche. En los individuos clasificados como mesenos, $15(13,9 \%)$ fueron de sexo femenino y $12(10,5 \%)$ de sexo masculino, donde en el grupo femenino hubo un individuo de etnia chileno-mapuche y dos en el 
Tabla I. Distribución del diámetro altura morfológica superior de la cara, según sexo.

\begin{tabular}{lcccc}
\hline Altura morfológica superior de la cara & Promedio & D.S. & Mínimo & Máximo \\
\hline Sexo & $\mathrm{mm}$ & $\mathrm{mm}$ & $\mathrm{mm}$ & $\mathrm{mm}$ \\
\hline Femenino & 56,6 & 3,71 & 44,4 & 69,9 \\
Masculino & 59,2 & 4,09 & 49,3 & 68,3 \\
\hline Total & 57,8 & 4,36 & 44,4 & 69,9 \\
\hline
\end{tabular}

Tabla II. Distribución del diámetro bicigomático máximo, según sexo.

\begin{tabular}{lcccc}
\hline Diámetro bicigomático máximo & Promedio & D.S. & Mínimo & Máximo \\
\hline Sexo & $\mathrm{mm}$ & & $\mathrm{mm}$ & $\mathrm{mm}$ \\
\hline Femenino & 121,5 & 6,92 & 109 & 152 \\
Masculino & 126,8 & 7,32 & 110 & 150 \\
\hline Total & 124,05 & 8,50 & 109 & 152 \\
\hline
\end{tabular}

grupo masculino. El caso de individuo hiperlepteno fue de sexo masculino y de etnia chileno-no mapuche (Fig. 1).

La distribución por sexos del ancho nasal se encuentra expresada en la Tabla IV y la altura del segmento nasal (N-Ns) en la Tabla V.

La distribución del grupo en estudio, respecto al índice nasal, se encuentra en la Tabla VI.

Tabla III. Distribución numérica y porcentual del índice facial superior.

\begin{tabular}{lcc}
\hline IFS & $\mathrm{N}^{\mathbf{o}}$ & $\%$ \\
\hline Hipereuriónico & Frecuencia & Porcentaje \\
Euriónico & 40 & 34,8 \\
Meseno & 47 & 40,9 \\
Lepteno & 27 & 23,5 \\
Hiperlepteno & 0 & 0 \\
\hline Total & 1 & 0,8 \\
\hline
\end{tabular}

Tabla IV. Distribución de la distancia del ancho nasal según sexo.

\begin{tabular}{lcccc}
\hline Sexo & $\begin{array}{c}\text { Promedio } \\
\mathrm{mm}\end{array}$ & $\begin{array}{c}\text { D.S. } \\
\mathrm{mm}\end{array}$ & $\begin{array}{c}\text { Mínimo } \\
\mathrm{mm}\end{array}$ & $\begin{array}{c}\text { Máximo } \\
\mathrm{mm}\end{array}$ \\
\hline Femenino & 46,1 & 6,34 & 38,5 & 49,9 \\
Masculino & 52,1 & 4,85 & 41,9 & 67,6 \\
\hline Ambos & 49,1 & 5,30 & 38,5 & 67,6 \\
\hline
\end{tabular}

Tabla V. Distribución de la Altura del Segmento Nasal según sexo.

\begin{tabular}{lcccc}
\hline & Promedio & D.S. & Mínimo & Máximo \\
\hline Sexo & $\mathrm{mm}$ & $\mathrm{mm}$ & $\mathrm{mm}$ & $\mathrm{mm}$ \\
\hline Femenino & 75,5 & 4,71 & 61,0 & 85,6 \\
Masculino & 78,3 & 4,85 & 67,1 & 90,9 \\
\hline Ambos & 76,8 & 4,76 & 61,0 & 90,9 \\
\hline
\end{tabular}

Tabla VI. Distribución numérica y porcentual del índice nasal.

\begin{tabular}{lcc}
\hline Índice nasal & Número & Porcentaje \\
\hline Hiperleptorrino (nariz muy angosta) & 4 & 3,5 \\
Leptorrino (nariz angosta) & 89 & 77,4 \\
Mesorrino (nariz mediana) & 20 & 17,4 \\
Camerrino (nariz ancha) & 2 & 1,7 \\
Hipercamerrino (nariz muy ancha) & 0 & 0 \\
Total & 115 & 100 \\
\hline
\end{tabular}

El índice nasal presentó un valor mínimo de 50,2 que clasificó a este individuo de sexo femenino, de etnia chilenono mapuche, como hiperleptorrino El IN máximo encontrado fue de 86,2, clasificando a este individuo como camerrino, siendo éste del sexo masculino y de etnia chileno-mapuche; el promedio de 63,9 determinó una población Leptorrina.

La distribución del IN en la población estudiada fue de 4 individuos hiperleptorrinos, lo que equivale al 3,5\%, con valores que fluctuaron entre 50,2 y 53,5, los cuales eran de sexo femenino y de etnia chileno-no mapuche.

Los individuos leptorrinos fueron 89 , equivalente al $77,4 \%$ de los casos, con valores que fluctuaron entre 55,2 y 69,9 de los cuales, $51(44,4 \%)$ correspondieron a sexo femenino, donde tres $(2,6 \%)$ eran de etnia chileno-mapuche; 38 individuos $(33,0 \%)$ eran de sexo masculino, uno $(0,9 \%)$ de etnia chileno-mapuche.

Se encontraron 20 individuos Mesorrinos (17,4\%), cuyos valores fluctuaron entre 70,0 y 77,7 siendo $4(3,5 \%)$ de sexo femenino y de etnia chileno-no mapuche, y, 16 $(13,9 \%)$ de sexo masculino, donde 2 eran de etnia chilenomapuche.

Como individuos camerrinos se encontraron 2 $(1,7 \%)$, con valores que fluctuaron entre 85,9 y 86,2 , ambos de sexo masculino, siendo uno de etnia chileno-mapuche Fig. 2. 


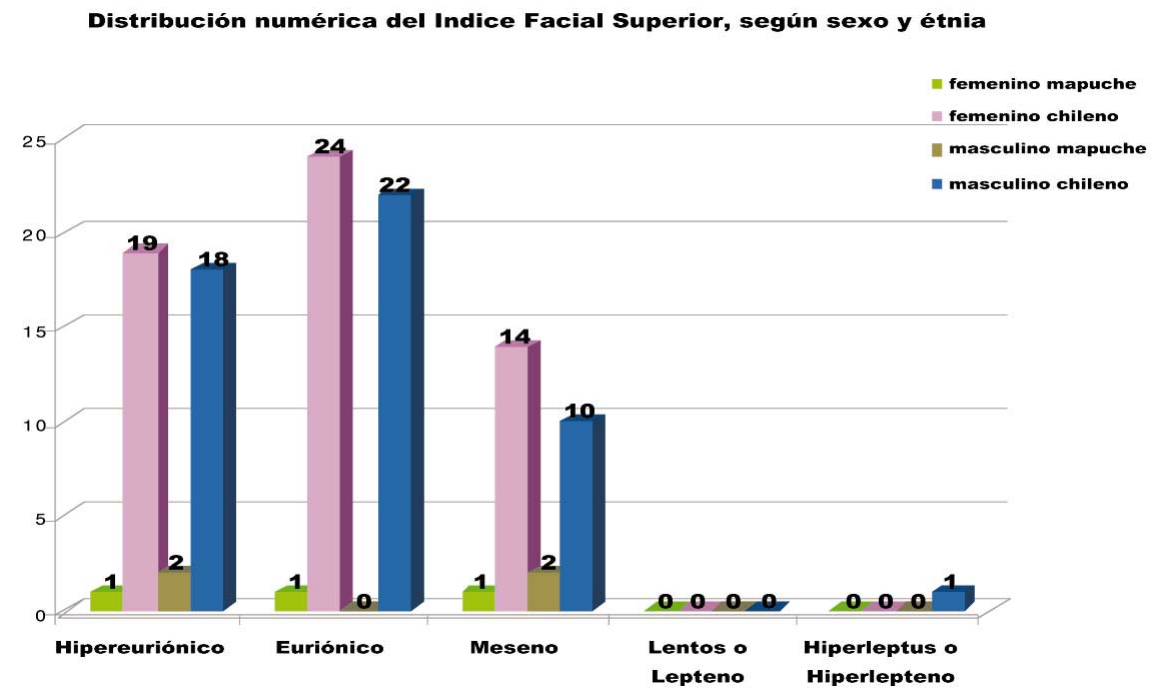

Fig. 1. Distribución numérica del índice facial superior según sexo y etnia.

\section{Distribución Numérica del Indice Nasal, según sexo y étnia}

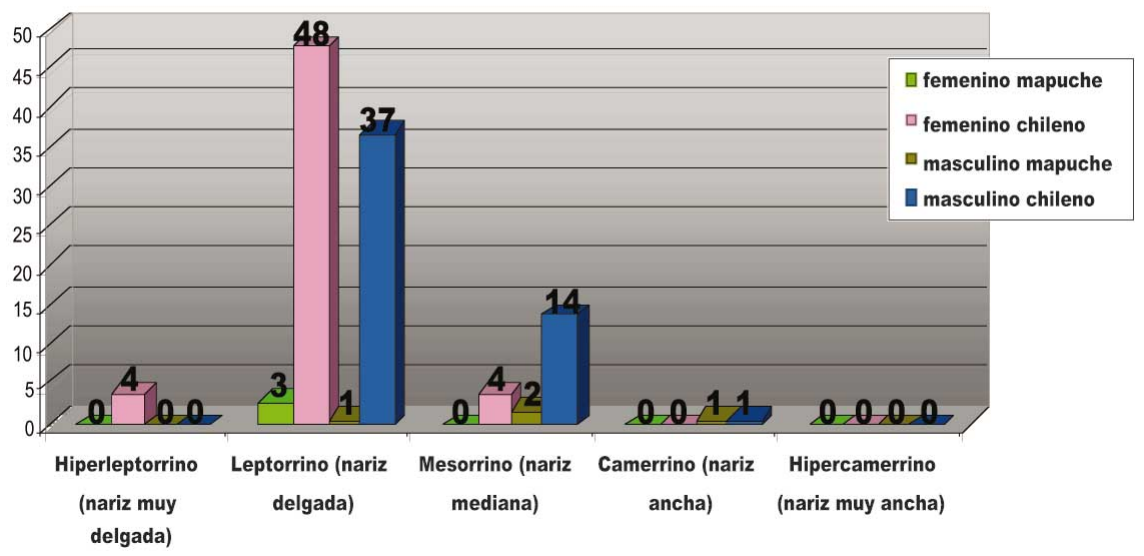

Fig. 2. Distribución numérica del índice nasal según sexo y etnia.

Al relacionar el índice facial superior y el índice nasal, no hubo asociación entre ambas variables ya que el valor de $\mathrm{P}$ fue de 0,45 . Al comparar los índices con la variable sexo aplicando prueba T-Test, fue estadísticamente significativa sólo con el IN (valor de $\mathrm{P}=0,0009$ ), en ambos sexos.

Al comparar los índices con la variable etnia con la prueba T-Test, concluimos que no hay evidencia estadística que permita inferir que están asociadas tanto para etnia chileno-mapuche como chileno-no mapuche (valor de P por sobre 0,05 ).

\section{DISCUSIÓN}

Las características faciales de los seres humanos le otorgan una individualidad especial, que permiten identificarlos dentro de un grupo determinado, así como también hay grupos de personas que tienen características que le diferencian de otros grupos que habitan el planeta. Son innumerables las variaciones que pueden presentar las partes que conforman la cara, aún dentro de un mismo individuo, si consideramos el lado derecho o el izquierdo. La simetría del rostro no es regla, pudiendo presentarse en algunos individuos una asimetría marcada. Se dice que el hombre es construido según el principio de la bilateralidad, pero en la realidad, no hay simetría perfecta porque no existe correspondencia exacta de todos los órganos (Dangelo \& Fattini, 1988).

El índice facial superior obtenido en nuestro estudio clasificó mayoritariamente a nuestro grupo de estudio como euriónicos e hipereuriónicos, diferente de lo obtenido por Goya et al. (1983), donde su población fue clasificada como hiperleptenos (promedio de 60,3 con DS 7,4). Esta diferencia llama la atención, ya que la población estudiada también es de nuestro país, pese a existir una distancia geográfica importante entre ambas regiones.

Nuestros resultados son similares a los obtenidos por del Sol (2006), en población Mapuche pura, de sexo masculino, quien obtuvo índices que clasificaron su grupo de estudio como euriónicos, de caras anchas. No se encontró otra investigación en individuos vivos de nuestro país, con la cual poder confrontar nuestros hallazgos.

Díaz et al. (2008) estudiando el tipo de cara del hombre andino merideño (Venezuela), en 60 estudiantes de la Facultad de Odontología, oriundos del Estado de Mérida, de edades entre 18 y 25 años, de ambos sexos, sin tratamiento de ortodoncia previo, determinaron que correspondían a individuos Euriónicos, (de acuerdo a Comas), presentando en la población masculina un promedio de 36,5 y en el sexo femenino 39,8 . 
Según la clasificación de Pospísil (1985) y Martin \& Saller, concordamos con los índices obtenidos por estos autores, ya que en nuestra investigación, el 75,7\% de las personas estudiadas corresponden a Hipereuriónicos y Euriónicos. Es interesante el hecho que el índice promedio en el sexo femenino del grupo de Mérida presente un valor mayor al masculino, característica no observada en nuestra población.

Al comparar los estudio de Fernández-Rico (1987) y Zavando et al., los índices informados por ellos son similares, clasificando a sus respectivos grupos como Mesenos, aún siendo individuos de dos continentes diferentes. Los valores encontrados en nuestra población difieren de los autores mencionados, donde el índice promedio fue de 46,8 , lo que corresponde al grupo de los Euriónicos. Cabe señalar que estos últimos estudios fueron realizados en cráneos secos y nuestro estudio lo fue en individuos vivos.

Así, el índice facial superior de nuestro grupo es concordante con los escasos estudios realizados en el país, los cuales se obtuvieron de etnias puras, lo que se explicaría por la conformación de la población local, la que también es propia de los pueblos xantodermos o amarillos.

La diferencia mayor entre los estudios mencionados, dice relación con el promedio del diámetro proporcionado por la altura morfológica de la parte superior de la cara, siendo mayor en la población de Brasil $(67,3 \mathrm{~mm}$, Zavando et al.) y menor en nuestros individuos $(57,9 \mathrm{~mm}$.). Esta diferencia podría deberse al tipo de individuos estudiados, ya que la mezcla con individuos de etnia negroide es alta en ese país. La cabeza de estos individuos tiene una altura mayor que la de nuestra población. Sin embargo el promedio del diámetro bicigomático es muy similar en estos tres grupos (diferencia de $0,39 \mathrm{~mm}$.).

En relación al índice nasal en nuestro estudio se clasificó a los individuos como Leptorrinos, tanto en el sexo femenino como en el masculino. En el estudio de Goya et al. realizado en Chile, se muestra en un cuadro de su artículo, valores que no coinciden con los descritos en el texto, ya que, de acuerdo a ellos, los individuos corresponderían a hipercamerrinos, (nariz muy ancha) con un índice promedio de 58,6 donde la población femenina presentó un índice de 58,3 (DS de 6,2) y la masculina un 59,0 (DS 8,6). Luego señalaron que los individuos de la población estudiada serían de narices estrechas (Leptorrinos), situación que entendemos como un problema de redacción. No se encontró otro estudio en Chile, en individuos vivos, con el cual poder confrontar nuestros resultados.

En España, Rebato \& Calderón (1988) estudiaron 400 individuos varones de, entre 19 y 65 años, clasificándolos como hiperleptorrinos.

Al realizar la comparación entre los estudios de Fernández- Rico y Zavando et al., tenemos índices diferentes, encontrándose en la Península Ibérica individuos hiperleptorrinos y en Brasil individuos camerrinos. Si bien no existe una explicación completa y consensuada con respecto a la relación que pudiera existir entre la forma de la nariz y la fisiología respiratoria, parece claro que una cavidad nasal alta y angosta, que proporciona una mayor superficie de mucosa, favorece el calentamiento y la humectación del aire inspirado y propicia la retención del calor y la humedad del aire espirado (Fernández-Rico) y existe evidencia de peso para atribuir ello a la adaptación al clima frío. El hecho es de que la adaptación al clima frío en la morfología nasal ha evolucionado con más fuerza en la relación entre altura y anchura, que en la superficie absoluta de la apertura nasal.

Al relacionar el índice facial superior y el índice nasal, aplicando la prueba de Chi-cuadrado, no existe asociación entre ambas variables ya que el valor de $\mathrm{P}$ es de 0,45 . $\mathrm{Al}$ comparar los índices con la variable sexo por prueba TTest, observamos que es estadísticamente significativa solo con el IN (valor de $\mathrm{P}=0,0009$ ), en ambos sexos, característica que está en concordancia con los expresado por autores como Marrodan (1995) y Fernández-Rico, quienes han indicado que es bien conocido que varones y mujeres muestran patrones diferentes en su crecimiento y desarrollo, donde la génesis masculina y femenina del sujeto condiciona una serie de pequeñas diferencias; por eso es importante establecer relaciones entre los rasgos definitorios de uno y otro sexo.

Al comparar los índices con la variable etnia con prueba T-Test, se aprecia que no hay evidencia estadística que permita inferir que están asociadas tanto para etnia chilenomapuche como chileno-no mapuche (valor de $\mathrm{P}$ por sobre $0,05)$. Por ello no concordamos con lo expresado por Brothwell (1987) en relación a establecer distancias biológicas entre grupos, pero creemos también que esto podría estar determinado por el hecho que nuestra población en estudio es pequeña.

Para tener un conocimiento más acabado de las poblaciones modernas, se hace necesario realizar investigaciones basadas preferentemente en individuos vivos, aportando y contribuyendo con datos de parámetros anatómicos y antropométricos, tanto a la Antropología Biológica o Física, como también a la antropología Forense y de este modo, entregar nuevos datos a los profesionales del área de la salud. 
BUSTAMANTE, F.; OLAVE, E. \& BINVIGNAT, O. Facial indices study in students of the Universidad de La Frontera, Chile. Int. J. Morphol., 29(4):1335-1340, 2011.

SUMMARY: Facial features of human beings give a special individuality, which can identify within a group, as well as groups of people with characteristics that differentiate them from other groups. In order to determine facial characteristics of our population, two facial indices were studied in adult individuals (18-35 years), of both sexes, students from the Universidad de La Frontera, Chile. The indexes investigated were the Superior Facial (SFI), which relates height of the upper face morphology with the maximum bizygomatic diameter and the nasal index (NI), which relates height and width nasal segment. The SFI average was 46.8 (SD 4.0), with maximum limits of 62.1 and minimum of 38.0, classifying individuals studied as Euryonics (wide face) in $41 \%$ of cases, as Hypereuryonics (face too wide) in 35\%, as Meson (medium face) in 23\% and as Lepton (narrow face) in 1\%. The NI average was 43.5 (SD 5.1), with maximum limits of 86.2 and minimum of 50.2, classifying individuals as leptorrhine (narrow nose) in $77.4 \%$ of cases, as mesorrhine (median nose) in $17.4 \%$ and catarrhine (wide nose) in 1.7\%. The results show that the majority of our population is of broad face and thin nose, data to complement the knowledge of the anthropometric features in our region.

KEY WORDS: Facial index; Nasal index; Young Adult; Chile.

\section{REFERENCIAS BIBLIOGRÁFICAS}

Bogin B. \& Collier S. Sexual dimorphism in relation to big-game hunting and economy in modern human populations. Am. J. Phys Anthropol. 91:485-504, 1993.

Brothwell, D.R. Desenterrando Huesos. La excavación, Tratamiento y Estudio de Restos del Esqueleto Humano. México, Fondo Cultura Económica, 1987.

Dangelo; J. \& Fattini, C. Anatomia Humana Sistemica e Segmentar. $2^{\mathrm{a}}$ ed. Rio de Janeiro, Atheneu, 1988.

Del Sol, M. Índices faciales en individuos mapuches Int. J. Morphol., 24(4):587-90, 2006.

Díaz, N.; Garcia, C.; Palacios, M.; Solórzano, E. \& Jarpa, P. Determinación del tipo de cara del hombre andino merideño, Estudio Morfoantropométrico del macizo facial. Bol. Antropol., 23:167-180, 2008.

Fernández-Rico, M. Crecimiento y evaluación del dimorfismo sexual en una población de Asturias. Actas V Cong. Soc. Esp. Antrop. Biol.:85-94. 1987.

Goya, T.; Cordero, C.; Ola, E. \& Álvarez, R. Índice nasal e índices faciales en individuos chilenos: morfología nasal. An. Anat. Norm;1(1):74-81, 1983.

Marrodán, M. D.; González-Montero de Espinosa, M. \& Prado, C. Antropología de la Nutrición. Técnicas, métodos y aplicaciones. Madrid, Noesis,1995. p. 277.

Martin, R. \& Saller, K. Lehrbuch der Anthropologie. Stuttgart. Gustav Fischer Verlag, 1957. p. 661.

Oliveros, G.; Durán, M.; Lorenzo, E. \& Martín, V. Tipos constitucionales. Arch. Méd.Camagüey;10(6), 2006, [en línea] Disponible en Internet: http://redalyc.uaemex.mx/src/inicio/ ArtPdfRed.jsp?iCve=211117906016. ISSN.
Pospísil, M. Manual de prácticas de antropología física. La Habana. Editorial del consejo nacional de Universidades, 1985. P. 208.

Rebato, E. \& Calderón R. Antropometría de la región céfalofacial del hombre vasco. Principe de Viana Sup. de Ciencias VI (6):261-267,1988.

Stini, A.W. Growth rates and sexual dimorphism in evolutionary perspective. The analysis of prehistoric diets. $1^{\mathrm{a}} \mathrm{ed}$, New York, Academic Press. Chapter, 1985. pp.123-156.

Zavando, M. D. A; Suazo, G. I. C \& Smith, R.L. ¿Es Posible la Determinación de la Afinidad Racial a Partir del Análisis Biométrico de Cráneos Humanos? Int. J. Morphol., 27(3):6438, 2009.

Dirección para correspondencia:

Prof. Dr. Enrique Olave

Facultad de Medicina

Universidad de La Frontera

Casilla 54-D

Temuco

CHILE

Email:eolave@ufro.cl

Recibido : 03-06-2011

Aceptado: 22-08-2011 